\title{
Common Fixed Points of Generalized Rational Type Cocyclic Mappings in Multiplicative Metric Spaces
}

\author{
Mujahid Abbas, ${ }^{1}$ Manuel De la Sen, ${ }^{2}$ and Talat Nazir ${ }^{3,4}$ \\ ${ }^{1}$ Department of Mathematics and Applied Mathematics, University Pretoria, Lynnwood Road, Pretoria 0002, South Africa \\ ${ }^{2}$ Institute of Research and Development of Process IIDP, Faculty of Science and Technology, University of the Basque Country, Spain \\ ${ }^{3}$ Division of Applied Mathematics, School of Education, Culture \& Communication, Mälardalen University, 72123 Västerås, Sweden \\ ${ }^{4}$ Department of Mathematics, COMSATS Institute of Information Technology, Abbottabad 22060, Pakistan
}

Correspondence should be addressed to Manuel De la Sen; manuel.delasen@ehu.es

Received 5 May 2015; Accepted 24 June 2015

Academic Editor: Irena Rachůnková

Copyright (C) 2015 Mujahid Abbas et al. This is an open access article distributed under the Creative Commons Attribution License, which permits unrestricted use, distribution, and reproduction in any medium, provided the original work is properly cited.

\begin{abstract}
The aim of this paper is to present fixed point result of mappings satisfying a generalized rational contractive condition in the setup of multiplicative metric spaces. As an application, we obtain a common fixed point of a pair of weakly compatible mappings. Some common fixed point results of pair of rational contractive types mappings involved in cocyclic representation of a nonempty subset of a multiplicative metric space are also obtained. Some examples are presented to support the results proved herein. Our results generalize and extend various results in the existing literature.
\end{abstract}

\section{Introduction and Preliminaries}

Fixed point theory provides the most important and traditional tools for proving the existence of solutions of many problems in both pure and applied mathematics. In metric fixed point theory, the interplay between contractive condition and the existence and uniqueness of a fixed point has been very strong and fruitful. The study of fixed points of mappings which satisfy certain contractive conditions has primary applications in the solution of differential and integral equations (see, e.g., [1-5]). Theorems dealing with fixed point of certain mappings inspired and motivated the development of many other important kinds of points like coincidence points, intersection points, sectional points, and so forth.

One of the basic and most widely applied fixed point theorems in mathematical analysis is "Banach Contraction Mapping Principle" (or Banach's Fixed Point Theorem). Kirk et al. [4] obtained some fixed point results for mappings satisfying cyclical contractive conditions. The Banach contraction mappings are continuous mappings, while cyclic contraction mappings need not be continuous. Păcurar and Rus [6] studied some fixed point results for cyclic weak contractions. Piątek [7] obtained some results on cyclic MeirKeeler contractions in metric spaces. Using fixed point result of weakly contractive map, Karapinar [3] established some interesting fixed point results for cyclic $\phi$-weak contraction mappings. Recently, Derafshpour et al. [8] obtained results on the existence of best proximity points of cyclic contractions. For more results in this direction, we refer to [9-15].

Banach contraction principle has been generalized either by extending the domain of the mapping or by considering a more general contractive condition on the mappings. Ozavsar and Cevikel [16] proved an analogous result to Banach contraction principle in the framework of multiplicative metric spaces. They also studied some topological properties of the relevant multiplicative metric space. Bashirov et al. [17] studied the concept of multiplicative calculus and proved a fundamental theorem of multiplicative calculus. They also illustrated the usefulness of multiplicative calculus with some interesting applications. Multiplicative calculus provides natural and straightforward way to compute the derivative of product and quotient of two functions [18]. It was shown that the multiplicative differential equations are more suitable than the ordinary differential equations in investigating some problems in economics and finance. 
Due to its operational simplicity and support to Newtonian calculus, it has attracted the attention of several researchers in the recent years. Furthermore, based on the definition of multiplicative absolute value function, they defined the multiplicative distance between two nonnegative real numbers and between two positive square matrices. This provided the basis for multiplicative metric spaces. Florack and van Assen [19] gave applications of multiplicative calculus in biomedical image analysis. He et al. [20] studied common fixed points for weak commutative mappings on a multiplicative metric space (see also [21]). Recently, Yamaod and Sintunavarat [22] obtained some fixed point results for generalized contraction mappings with cyclic $(\alpha, \beta)$-admissible mapping in multiplicative metric spaces.

In this paper, we obtain fixed point result of a generalized rational contractive mapping in the framework of multiplicative metric spaces. Employing this result, a common fixed point of a pair of weakly compatible mappings is obtained. We study the sufficient conditions for the existence of common fixed points of pair of rational contractive types mappings involved in cocyclic representation of a nonempty subset of a multiplicative metric space. Our results generalize and extend comparable results in [3].

By $\mathbb{R}, \mathbb{R}^{+}, \mathbb{R}_{n}^{+}$, and $\mathbb{N}$ we denote the set of all real numbers, the set of all nonnegative real numbers, the set of all $n$-tuples of positive real numbers, and the set of all natural numbers, respectively.

Consistent with $[16,17]$, the following definitions and results will be needed in the sequel.

Definition 1 (see [17]). Let $X$ be a nonempty set. A mapping $d: X \times X \rightarrow \mathbb{R}^{+}$is said to be a multiplicative metric on $X$ if for any $x, y, z \in X$, the following conditions hold:

(i) $d(x, y) \geq 1$ and $d(x, y)=1$ if and only if $x=y$.

(ii) $d(x, y)=d(y, x)$.

(iii) $d(x, y) \leq d(x, z) \cdot d(z, y)$.

The pair $(X, d)$ is called a multiplicative metric space.

We define absolute valued function that includes the negative real numbers in its domain.

Definition 2. A multiplicative absolute value function $|\cdot|$ : $\mathbb{R} \rightarrow \mathbb{R}^{+}$is defined as

$$
|x|=\left\{\begin{aligned}
x & \text { if } x \geq 1, \\
\frac{1}{x} & \text { if } x \in(0,1), \\
1 & \text { if } x=0, \\
-\frac{1}{x} & \text { if } x \in(-1,0), \\
-x & \text { if } x \leq-1 .
\end{aligned}\right.
$$

Using the definition of multiplicative absolute value function, we can prove the following proposition.

Proposition 3. For arbitrary $x, y \in \mathbb{R}^{+}$, the multiplicative absolute value function $|\cdot|: \mathbb{R}^{+} \rightarrow \mathbb{R}^{+}$satisfies the following:
(1) $|x| \geq 1$.
(2) $x \leq|x|$.
(3) $1 /|x| \leq x$ if $x>0$ and $x \leq 1 /|x|$ if $x \leq 0$.
(4) $|x \cdot y| \leq|x||y|$.

Example 4 (see [16]). Let $X=\mathbb{R}_{n}^{+}$. Then

$$
\begin{aligned}
& d_{1}(x, y)=\left|\frac{x_{1}}{y_{1}}\right| \cdot\left|\frac{x_{2}}{y_{2}}\right| \ldots \ldots\left|\frac{x_{n}}{y_{n}}\right|, \\
& d_{2}(x, y)=\max \left\{\left|\frac{x_{1}}{y_{1}}\right|,\left|\frac{x_{2}}{y_{2}}\right|, \ldots,\left|\frac{x_{n}}{y_{n}}\right|\right\}
\end{aligned}
$$

define multiplicative metrices on $X$, where $x=\left(x_{1}, x_{2}, \ldots\right.$, $\left.x_{n}\right)$ and $y=\left(y_{1}, y_{2}, \ldots, y_{n}\right) \in \mathbb{R}_{n}^{+}$.

Definition 5 (see [16]). Let $(X, d)$ be a multiplicative metric space, $x_{0}$ an arbitrary point in $X$, and $\varepsilon>1$. A multiplicative open ball $B\left(x_{0}, \varepsilon\right)$ of radius $\varepsilon$ centered at $x_{0}$ is the set $\{z \in X$ : $\left.d\left(z, x_{0}\right)<\varepsilon\right\}$.

A sequence $\left\{x_{n}\right\}$ in a multiplicative metric space $(X, d)$ is said to be multiplicative convergent to some point $x \in X$ if, for any given $\varepsilon>1$, there exists $n_{0} \in \mathbb{N}$ such that $x_{n} \in B(x, \varepsilon)$ for all $n \geq n_{0}$. If $\left\{x_{n}\right\}$ converges to $x$, we write $x_{n} \rightarrow x$ as $n \rightarrow \infty$

Definition 6 (see [16]). A sequence $\left\{x_{n}\right\}$ in a multiplicative metric space $(X, d)$ is multiplicative convergent to $x$ in $X$ if and only if $d\left(x_{n}, x\right) \rightarrow 1$ as $n \rightarrow \infty$.

Definition 7. Let $\left(X, d_{X}\right)$ and $\left(Y, d_{Y}\right)$ be two multiplicative metric spaces and $x_{0}$ an arbitrary but fixed element of $X$. A mapping $f: X \rightarrow Y$ is said to be multiplicative continuous at $x_{0}$ if and only if $x_{n} \rightarrow x_{0}$ in $\left(X, d_{X}\right)$ implies that $f\left(x_{n}\right) \rightarrow$ $f\left(x_{0}\right)$ in $\left(Y, d_{Y}\right)$. That is, given arbitrary $\varepsilon>1$, there exists $\delta>1$ which depends on $x_{0}$ and $\varepsilon$ such that $d_{Y}\left(f x, f x_{0}\right)<\varepsilon$ for all those $x$ in $X$ for which $d_{X}\left(x, x_{0}\right)<\delta$.

Definition 8 (see [16]). A sequence $\left\{x_{n}\right\}$ in a multiplicative metric space $(X, d)$ is said to be multiplicative Cauchy sequence if, for any $\varepsilon>1$, there exists $n_{0} \in \mathbb{N}$ such that $d\left(x_{n}, x_{m}\right)<\varepsilon$ for all $m, n \geq n_{0}$.

A multiplicative metric space $(X, d)$ is said to be complete if every multiplicative Cauchy sequence $\left\{x_{n}\right\}$ in $X$ is multiplicative convergent in $X$.

Theorem 9 (see [16]). A sequence $\left\{x_{n}\right\}$ in a multiplicative metric space $(X, d)$ is multiplicative Cauchy if and only if $d\left(x_{n}, x_{m}\right) \rightarrow 1$ as $n, m \rightarrow \infty$.

Example 10. Let $X=C^{*}[a, b]$ be the collection of all realvalued multiplicative continuous functions over $[a, b] \subseteq \mathbb{R}^{+}$ with the multiplicative metric $d$ defined by

$$
d(f, g)=\sup _{x \in[a, b]}\left|\frac{f(x)}{g(x)}\right| \quad \text { for arbitrary } f, g \in X
$$

where $|\cdot|: \mathbb{R}^{+} \rightarrow \mathbb{R}^{+}$is a multiplicative absolute value function. Then $\left(C^{*}[a, b], d\right)$ is complete. 
Recall that if $f$ and $g$ are two self-maps on a set $X$ and $u=f x=g x$ for some $x$ in $X$, then $x$ is called a coincidence point of $f$ and $g$, and $u$ is called a point of coincidence of $f$ and g.

Definition 11 (see [23]). Two self-maps $f$ and $g$ on a nonempty set $X$ are called weakly compatible if they commute at their coincidence point.

We will also need the following proposition from [23].

Proposition 12. Let $f$ and $g$ be weakly compatible self-maps on a set $X$. If $f$ and $g$ have a unique point of coincidence $w=$ $f x=g x$, then $w$ is the unique common fixed point of $f$ and $g$.

Definition 13 (see [12]). Let $\left\{X_{i}: i=1,2, \ldots, p\right\}$ be a finite collection of nonempty subsets of a set $X$, where $p$ is some positive integer and $f, g: X \rightarrow X$. The set $X$ is said to have a cocyclic representation with respect to the collection $\left\{X_{i}\right.$ : $i=1,2, \ldots, p\}$ and a pair $(f, g)$ if

(1) $X=\cup_{i=1}^{p} X_{i}$;

(2) $f\left(X_{1}\right) \subseteq g\left(X_{2}\right), \ldots, f\left(X_{p-1}\right) \subseteq g\left(X_{p}\right), f\left(X_{p}\right) \subseteq$ $g\left(X_{1}\right)$.

Definition 14. The control functions $\psi$ and $\varphi$ are defined as follows:

(i) $\psi:[1, \infty) \rightarrow[1, \infty)$ is a continuous nondecreasing function with $\psi(t)=1$ if and only if $t=1$.

(ii) $\varphi:[1, \infty) \rightarrow[1, \infty)$ is a lower semicontinuous function with $\varphi(t)=1$ if and only if $t=1$.

\section{Main Results}

In this section, we obtain several fixed and common fixed point results of self-maps satisfying certain generalized contractive conditions in the framework of multiplicative metric space.

We start with the following result.

Theorem 15. Let $(X, d)$ be a complete multiplicative metric space and $f: X \rightarrow X$. Suppose that there exist control functions $\psi$ and $\varphi$ such that

$$
\psi(d(f x, f y)) \leq \frac{\psi\left(M_{f}(x, y)\right)}{\varphi\left(M_{f}(x, y)\right)},
$$

for any $x, y \in X$, where

$$
\begin{gathered}
M_{f}(x, y)=\max \{d(x, y), d(f x, x), d(f y, y), \\
\left.(d(f x, y) \cdot d(f y, x))^{1 / 2}\right\} .
\end{gathered}
$$

Then $f$ has a unique fixed point.

Proof. Let $x_{0}$ be a given point in $X$. Define a sequence $\left\{x_{n}\right\}$ in $X$ as $x_{n}=f^{n} x_{0}$ or equivalently as $x_{n+1}=f x_{n}$ for $n \in \mathbb{N}$. If $x_{k}=x_{k+1}$ for some $k$, then we have $f x_{k}=x_{k+1}=x_{k}$ and the result follows. Assume that $x_{n+1} \neq x_{n}$, for all $n \in \mathbb{N}$; that is, $M_{f}\left(x_{n+1}, x_{n}\right)>1$ for all $n \in \mathbb{N}$. From (4), we have

$$
\begin{aligned}
\psi\left(d\left(x_{n+2}, x_{n+1}\right)\right) & =\psi\left(d\left(f x_{n+1}, f x_{n}\right)\right) \\
& \leq \frac{\psi\left(M_{f}\left(x_{n+1}, x_{n}\right)\right)}{\varphi\left(M_{f}\left(x_{n+1}, x_{n}\right)\right)},
\end{aligned}
$$

where

$$
\begin{aligned}
& M_{f}\left(x_{n+1}, x_{n}\right)=\max \left\{d\left(x_{n+1}, x_{n}\right), d\left(f x_{n+1}, x_{n+1}\right),\right. \\
& \left.d\left(f x_{n}, x_{n}\right),\left(d\left(f x_{n+1}, x_{n}\right) \cdot d\left(f x_{n}, x_{n+1}\right)\right)^{1 / 2}\right\} \\
& \quad=\max \left\{d\left(x_{n+1}, x_{n}\right), d\left(x_{n+2}, x_{n+1}\right), d\left(x_{n+1}, x_{n}\right),\right. \\
& \left.\left(d\left(x_{n+2}, x_{n}\right) \cdot d\left(x_{n+1}, x_{n+1}\right)\right)^{1 / 2}\right\} \\
& \quad=\max \left\{d\left(x_{n+1}, x_{n}\right), d\left(x_{n+2}, x_{n+1}\right), d\left(x_{n+2}, x_{n}\right)^{1 / 2}\right\} \\
& \quad \leq \max \left\{d\left(x_{n+1}, x_{n}\right), d\left(x_{n+2}, x_{n+1}\right),\right. \\
& \left.\quad\left(d\left(x_{n+2}, x_{n+1}\right) \cdot d\left(x_{n+1}, x_{n}\right)\right)^{1 / 2}\right\} \\
& \quad \leq \max \left\{d\left(x_{n+1}, x_{n}\right), d\left(x_{n+2}, x_{n+1}\right),\right. \\
& \left.\quad\left(\frac{d\left(x_{n+2}, x_{n+1}\right)+d\left(x_{n+1}, x_{n}\right)}{2}\right)\right\} \\
& \quad=\max \left\{d\left(x_{n+1}, x_{n}\right), d\left(x_{n+2}, x_{n+1}\right)\right\} .
\end{aligned}
$$

If $M_{f}\left(x_{k+1}, x_{k}\right) \leq d\left(x_{k+2}, x_{k+1}\right)$ for some $k \in \mathbb{N}$, then $\psi\left(d\left(x_{k+2}, x_{k+1}\right)\right) \leq \psi\left(d\left(x_{k+2}, x_{k+1}\right)\right) / \varphi\left(M_{f}\left(x_{k+1}, x_{k}\right)\right)$ implies $\varphi\left(M_{f}\left(x_{k+1}, x_{k}\right)\right) \leq 1$, a contradiction. Hence $M_{f}\left(x_{n+1}, x_{n}\right) \leq$ $d\left(x_{n+1}, x_{n}\right)$ for all $n \in \mathbb{N}$. Also, $d\left(x_{n+1}, x_{n}\right) \leq M_{f}\left(x_{n+1}, x_{n}\right)$ for all $n \in \mathbb{N}$ and hence $M_{f}\left(x_{n+1}, x_{n}\right)=d\left(x_{n+1}, x_{n}\right)$ for all $n \in \mathbb{N}$. Thus, we have

$$
\begin{aligned}
\psi\left(d\left(x_{n+2}, x_{n+1}\right)\right) & \leq \frac{\psi\left(d\left(x_{n+1}, x_{n}\right)\right)}{\varphi\left(d\left(x_{n+1}, x_{n}\right)\right)} \\
& <\psi\left(d\left(x_{n+1}, x_{n}\right)\right)
\end{aligned}
$$

which implies that

$$
d\left(x_{n+2}, x_{n+1}\right) \leq d\left(x_{n+1}, x_{n}\right)
$$

for all $n \in \mathbb{N}$. Thus $\left\{d\left(x_{n+1}, x_{n}\right)\right\}$ is (strictly) decreasing of positive real numbers. Consequently, there exists $c \geq 1$ such that $\left\{d\left(x_{n+1}, x_{n}\right)\right\}$ converges to $c$. Suppose that $c>1$. Now

$$
\psi\left(d\left(x_{n+2}, x_{n+1}\right)\right) \leq \frac{\psi\left(M_{f}\left(x_{n+1}, x_{n}\right)\right)}{\varphi\left(M_{f}\left(x_{n+1}, x_{n}\right)\right)},
$$

and lower semicontinuity of $\varphi$ gives that

$$
\begin{aligned}
& \limsup _{n \rightarrow \infty} \psi\left(d\left(x_{n+2}, x_{n+1}\right)\right) \\
& \quad \leq \frac{\limsup _{n \rightarrow \infty} \psi\left(M_{f}\left(x_{n+1}, x_{n}\right)\right)}{\liminf _{n \rightarrow \infty} \varphi\left(M_{f}\left(x_{n+1}, x_{n}\right)\right)},
\end{aligned}
$$


which implies that $\psi(c) \leq \psi(c) / \varphi(c)<\psi(c)$, a contradiction as $\varphi(c)>1$. Therefore, $c=1$; that is, $\lim _{n \rightarrow \infty} d\left(x_{n+1}, x_{n}\right)=1$.

Now, we claim that $\lim _{n, m \rightarrow \infty} d\left(x_{n}, x_{m}\right)=1$. If not, then there exist $\varepsilon>1$ and sequences $\left\{n_{k}\right\},\left\{m_{k}\right\}$ in $\mathbb{N}$, with $n_{k}>m_{k} \geq k$, such that $d\left(x_{n_{k}}, x_{m_{k}}\right) \geq \varepsilon$ for all $k \in \mathbb{N}$. Without any loss of generality, we can assume that $d\left(x_{n_{k}}, x_{m_{k}-1}\right)<\varepsilon$. Since $\left\{d\left(x_{m_{k}-1}, x_{m_{k}}\right)\right\}$ is a subsequence of convergent sequence $\left\{d\left(x_{n-1}, x_{n}\right)\right\} \rightarrow 1$ as $n \rightarrow \infty$, then $\left\{d\left(x_{m_{k}-1}, x_{m_{k}}\right)\right\} \rightarrow 1$ as $k \rightarrow \infty$. Now

$$
\varepsilon \leq d\left(x_{m_{k}}, x_{n_{k}}\right) \leq d\left(x_{n_{k}}, x_{m_{k}-1}\right) \cdot d\left(x_{m_{k}-1}, x_{m_{k}}\right)
$$

implies that

$$
\lim _{k \rightarrow \infty} d\left(x_{m_{k}}, x_{n_{k}}\right)=\varepsilon
$$

From (13) and inequality $d\left(x_{m_{k}}, x_{n_{k}}\right) \leq d\left(x_{m_{k}}, x_{m_{k}-1}\right)$. $d\left(x_{m_{k}-1}, x_{n_{k}}\right)$, it follows that $\varepsilon \leq \lim _{k \rightarrow \infty} d\left(x_{m_{k}-1}, x_{n_{k}}\right)$. Also, the inequality $d\left(x_{m_{k}-1}, x_{n_{k}}\right) \leq d\left(x_{m_{k}-1}, x_{m_{k}}\right) \cdot d\left(x_{m_{k}}, x_{n_{k}}\right)$ and (13) give that $\lim _{k \rightarrow \infty} d\left(x_{m_{k}-1}, x_{n_{k}}\right) \leq \varepsilon$, and hence we have

$$
\lim _{k \rightarrow \infty} d\left(x_{m_{k}-1}, x_{n_{k}}\right)=\varepsilon .
$$

Equation (14) and inequality $d\left(x_{m_{k}-1}, x_{n_{k}}\right) \leq d\left(x_{m_{k}-1}, x_{n_{k}+1}\right)$. $d\left(x_{n_{k}+1}, x_{n_{k}}\right)$ imply that $\varepsilon \leq \lim _{k \rightarrow \infty} d\left(x_{m_{k}-1}, x_{n_{k}+1}\right)$, while inequality $d\left(x_{m_{k}-1}, x_{n_{k}+1}\right) \leq d\left(x_{m_{k}-1}, x_{n_{k}}\right) \cdot d\left(x_{n_{k}}, x_{n_{k}+1}\right)$ and (14) imply that $\lim _{k \rightarrow \infty} d\left(x_{m_{k}-1}, x_{n_{k}+1}\right) \leq \varepsilon$, and hence we have

$$
\lim _{k \rightarrow \infty} d\left(x_{m_{k}-1}, x_{n_{k}+1}\right)=\varepsilon
$$

From (13) and $d\left(x_{m_{k}}, x_{n_{k}}\right) \leq d\left(x_{m_{k}}, x_{n_{k}+1}\right) \cdot d\left(x_{n_{k}+1}, x_{n_{k}}\right)$, we have $\varepsilon \leq \lim _{k \rightarrow \infty} d\left(x_{m_{k}}, x_{n_{k}+1}\right)$, and the inequality $d\left(x_{m_{k}}, x_{n_{k}+1}\right) \leq d\left(x_{m_{k}}, x_{n_{k}}\right) \cdot d\left(x_{n_{k}}, x_{n_{k}+1}\right)$ and (13) give that $\lim _{k \rightarrow \infty} d\left(x_{m_{k}}, x_{n_{k}+1}\right) \leq \varepsilon$. So

$$
\lim _{k \rightarrow \infty} d\left(x_{m_{k}}, x_{n_{k}+1}\right)=\varepsilon .
$$

As

$$
\begin{aligned}
& M_{f}\left(x_{n_{k}}, x_{m_{k}-1}\right)=\max \left\{d\left(x_{n_{k}}, x_{m_{k}-1}\right),\right. \\
& d\left(f x_{n_{k}}, x_{n_{k}}\right), d\left(f x_{m_{k}-1}, x_{m_{k}-1}\right), \\
& \left.\left(d\left(x_{n_{k}}, f x_{m_{k}-1}\right) \cdot d\left(x_{m_{k}-1}, f x_{n_{k}}\right)\right)^{1 / 2}\right\} \\
& =\max \left\{d\left(x_{n_{k}}, x_{m_{k}-1}\right), d\left(x_{n_{k}+1}, x_{n_{k}}\right),\right. \\
& \left.d\left(x_{m_{k}}, x_{m_{k}-1}\right),\left(d\left(x_{n_{k}}, x_{m_{k}}\right) \cdot\left(x_{m_{k}-1}, x_{n_{k}+1}\right)\right)^{1 / 2}\right\},
\end{aligned}
$$

we have $\lim _{k \rightarrow \infty} M_{f}\left(x_{n_{k}}, x_{m_{k}-1}\right)=\max \{\varepsilon, 1,1, \varepsilon\}=\varepsilon$. From (4), it follows that

$$
\begin{aligned}
\psi\left(d\left(x_{n_{k}+1}, x_{m_{k}}\right)\right) & =\psi\left(d\left(f x_{n_{k}}, f x_{m_{k}-1}\right)\right) \\
& \leq \frac{\psi\left(M_{f}\left(x_{n_{k}}, x_{m_{k}-1}\right)\right)}{\varphi\left(M_{f}\left(x_{n_{k}}, x_{m_{k}-1}\right)\right)} .
\end{aligned}
$$

Taking upper limit as $k \rightarrow \infty$ implies that $\psi(\varepsilon) \leq$ $\psi(\varepsilon) / \varphi(\varepsilon)<\psi(\varepsilon)$, a contradiction as $\varepsilon>1$. Thus, we obtain that $\lim _{n, m \rightarrow \infty} d\left(x_{n}, x_{m}\right)=1$, and hence $\left\{x_{n}\right\}$ is a multiplicative Cauchy sequence in $(X, d)$. Next, we assume there exists a point $u \in X$ such that $\lim _{n \rightarrow \infty} d\left(u, x_{n}\right)=1$; equivalently,

$$
\lim _{n, m \rightarrow \infty} d\left(x_{n}, x_{m}\right)=\lim _{n \rightarrow \infty} d\left(x_{n}, u\right)=1 \text {. }
$$

Note that

$$
\begin{aligned}
& d(f u, u) \leq M_{f}\left(u, x_{n}\right)=\max \left\{d\left(u, x_{n}\right), d(f u, u),\right. \\
& \left.d\left(f x_{n}, x_{n}\right),\left(d\left(u, f x_{n}\right) \cdot d\left(x_{n}, f u\right)\right)^{1 / 2}\right\} \\
& =\max \left\{d\left(u, x_{n}\right), d(f u, u), d\left(x_{n+1}, x_{n}\right),\right. \\
& \left.\left(d\left(u, x_{n+1}\right) \cdot d\left(x_{n}, f u\right)\right)^{1 / 2}\right\} \leq \max \left\{d\left(u, x_{n}\right),\right. \\
& d(f u, u), d\left(x_{n+1}, x_{n}\right), \\
& \left.\left(d\left(u, x_{n+1}\right) \cdot d\left(x_{n}, u\right) \cdot d(u, f u)\right)^{1 / 2}\right\} .
\end{aligned}
$$

Taking limit as $n \rightarrow \infty$, we conclude that $\lim _{n \rightarrow \infty} M_{f}\left(u, x_{n}\right)$ $=d(f u, u)$. Hence

$$
\psi\left(d\left(f u, f x_{n+1}\right)\right) \leq \frac{\psi\left(M_{f}\left(u, x_{n}\right)\right)}{\varphi\left(M_{f}\left(u, x_{n}\right)\right)}
$$

on taking upper limit as $n \rightarrow \infty$ implies that

$$
\psi(d(f u, u)) \leq \frac{\psi(d(f u, u))}{\varphi(d(f u, u))}
$$

which further implies that $f u=u$.

To prove the uniqueness of fixed point of $f$, assume on the contrary that $f v=v$ and $f w=w$ with $v \neq w$. Note that

$$
\psi(d(v, w))=\psi(d(f v, f w)) \leq \frac{\psi\left(M_{f}(v, w)\right)}{\varphi\left(M_{f}(v, w)\right)}
$$

where

$$
\begin{aligned}
& M_{f}(v, w)=\max \{d(v, w), d(f v, v), d(f w, w), \\
& \left.(d(f v, w) \cdot d(f w, v))^{1 / 2}\right\}=\max \{d(v, w), \\
& \left.d(v, v), d(w, w),(d(v, w) \cdot d(w, v))^{1 / 2}\right\}=d(v, w) .
\end{aligned}
$$

From (23) it follows that

$$
\begin{aligned}
\psi(d(v, w)) & =\psi(d(f v, f w)) \leq \frac{\psi(d(v, w))}{\varphi(d(v, w))} \\
& <\psi(d(v, w)),
\end{aligned}
$$

a contradiction as $d(v, w)>1$. Hence $v=w$. 
Corollary 16. Let $(X, d)$ be a complete multiplicative metric space and $f: X \rightarrow X$. Suppose that there exist control functions $\psi$ and $\varphi$ such that

$$
\psi\left(d\left(f^{n} x, f^{n} y\right)\right) \leq \frac{\psi\left(M_{f^{n}}(x, y)\right)}{\varphi\left(M_{f^{n}}(x, y)\right)},
$$

for any $x, y \in X$ and $n \in \mathbb{N}$ where

$$
\begin{gathered}
M_{f^{n}}(x, y)=\max \left\{d(x, y), d\left(f^{n} x, x\right), d\left(f^{n} y, y\right),\right. \\
\left.\left(d\left(f^{n} x, y\right) \cdot d\left(f^{n} y, x\right)\right)^{1 / 2}\right\} .
\end{gathered}
$$

Then $f$ has a unique fixed point $u$ and $f^{n} u=f u$.

Proof. Set $T=f^{n}$. From Theorem 15, $T$ has a unique fixed point $u$. Now $f(u)=f(T u)=f^{n+1}(u)=f^{n}(f u)=T(f u)$ which implies that $f u$ is also a fixed point of T. By the uniqueness of fixed point of $T$, we have $f u=u$.

Now, we recall the following lemma from [24].

Lemma 17. Let $X$ be a nonempty set and $f: X \rightarrow X$. Then there exists a subset $E \subseteq X$ such that $f(E)=f(X)$ and $f$ : $E \rightarrow X$ is one-to-one.

Theorem 18. Let $(X, d)$ be a multiplicative metric space and $f, g: X \rightarrow X$. Suppose that there exist control functions $\psi$ and $\varphi$ such that

$$
\psi(d(f x, f y)) \leq \frac{\psi(M(g x, g y))}{\varphi(M(g x, g y))}
$$

holds for any $x, y \in X$, where

$$
\begin{aligned}
& M(x, y)=\max \{d(x, y), d(f x, g x), d(f y, g y), \\
& \left.(d(f x, g y) \cdot d(f y, g x))^{1 / 2}\right\} .
\end{aligned}
$$

If $g(X)$ is a complete subspace of $X$, then $f$ and $g$ have a unique coincidence point in X. Moreover, if $f$ and $g$ are weakly compatible, then $f$ and $g$ have at most one common fixed point.

Proof. By Lemma 17, there exists $E \subseteq X$ such that $g(E)=$ $g(X)$ and $g: E \rightarrow X$ is one-to-one. Define a map $h: g(E) \rightarrow$ $g(E)$ by $h(g x)=f x$. Since $g$ is one-to-one on $E, h$ is well defined. Note that

$$
\psi(d(h(g x), h(g y))) \leq \frac{\psi(M(g x, g y))}{\varphi(M(g x, g y))},
$$

for all $g x, g y \in g(E)$ where

$$
\begin{aligned}
& M(g x, g y)=\max \{d(g x, g y), d(h(g x), g x), \\
& \quad d(h(g y), g y),(d(h(g x), g y) \cdot d(h(g y), g x))\} .
\end{aligned}
$$

Since $g(E)=g(X)$ is complete, by Theorem 15, there exists $x_{0} \in X$ such that $h\left(g x_{0}\right)=g x_{0}=f x_{0}$. Hence, $f$ and $g$ have a unique point of coincidence. From Proposition 12, $f$ and $g$ have a unique common fixed point.

\section{Cocyclic Contractions}

Now we obtain common fixed point results for self-maps satisfying certain cocyclic contractions defined on a multiplicative metric space. We start with the following.

Theorem 19. Let $(X, d)$ be a multiplicative metric space, $A_{1}, A_{2}, \ldots, A_{p}$ nonempty closed subsets of $X$, and $Y=\cup_{i=1}^{P} A_{i}$. Suppose that $f, g: Y \rightarrow Y$ are such that

(a) $Y$ has a cocyclic representation with respect to pair $(f, g)$ and the collection $\left\{A_{i}: i=1,2, \ldots, p\right\}$;

(b) there exists control functions $\psi$ and $\varphi$ such that, for any $(x, y) \in A_{i} \times A_{i+1}, i=1,2, \ldots, p$, the following,

$$
\psi(d(f x, f y)) \leq \frac{\psi\left(M_{f, g}(x, y)\right)}{\varphi\left(M_{f, g}(x, y)\right)},
$$

holds where

$$
\begin{gathered}
M_{f, g}(x, y)=\max \{d(g x, g y), d(f x, g x), \\
\left.d(f y, g y),(d(f x, g y) \cdot d(f y, g x))^{1 / 2}\right\}
\end{gathered}
$$

with $A_{p+1}=A_{1}$.

If $g\left(A_{i}\right)$ is complete subspace of $X$ for each $i \in\{1,2, \ldots, p\}$, then $f$ and $g$ have a unique coincidence point $z \in \cap_{i=1}^{p} g\left(A_{i}\right)=$ $Z$ provided that $f(Z) \subseteq g(Z) \subseteq Z$ and $g(Z)$ is closed. Moreover, if $f$ and $g$ are weakly compatible, then $f$ and $g$ have at most one common fixed point.

Proof. Let $x_{0}$ be a given point in $\cup_{i=1}^{p} A_{i}$. Then there exists $i_{0} \in\{1,2, \ldots, p\}$ such that $x_{0} \in A_{i_{0}}$. Choose a point $x_{1}$ in $A_{i_{0}+1}$ such that $f\left(x_{0}\right)=g\left(x_{1}\right)$. This can be done because $f\left(A_{i_{0}}\right) \subseteq g\left(A_{i_{0}+1}\right)$. Continuing this process, for $n>0$, there exists $i_{n} \in\{1,2, \ldots, p\}$ such that having chosen $x_{n}$ in $A_{i_{n}}$, we obtain $x_{n+1}$ in $A_{i_{n}+1}$ such that $f\left(x_{n}\right)=g\left(x_{n+1}\right)$. If, for some $n_{0} \geq 0$, we have $f\left(x_{n_{0}}\right)=f\left(x_{n_{0}+1}\right)$, then $f\left(x_{n_{0}}\right)=g\left(x_{n_{0}}\right)$ implies that $x_{n_{0}}$ is the coincidence point of $f$ and $g$. Assume that $d\left(f\left(x_{n}\right), f\left(x_{n+1}\right)\right)>1$ for all $n$. From (b), we have

$$
\begin{aligned}
\psi\left(d\left(g x_{n+1}, g x_{n+2}\right)\right) & =\psi\left(d\left(f x_{n}, f x_{n+1}\right)\right) \\
& \leq \frac{\psi\left(M_{f, g}\left(x_{n}, x_{n+1}\right)\right)}{\varphi\left(M_{f, g}\left(x_{n}, x_{n+1}\right)\right)} \\
& \leq \psi\left(M_{f, g}\left(x_{n}, x_{n+1}\right)\right),
\end{aligned}
$$

where

$$
\begin{aligned}
& M_{f, g}\left(x_{n}, x_{n+1}\right)=\max \left\{d\left(g x_{n}, g x_{n+1}\right), d\left(f x_{n}, g x_{n}\right),\right. \\
& d\left(f x_{n+1}, g x_{n+1}\right),
\end{aligned}
$$




$$
\begin{aligned}
& \left.\left(d\left(f x_{n}, g x_{n+1}\right) \cdot d\left(f x_{n+1}, g x_{n}\right)\right)^{1 / 2}\right\} \\
& =\max \left\{d\left(g x_{n}, g x_{n+1}\right), d\left(g x_{n+1}, g x_{n}\right),\right. \\
& d\left(g x_{n+2}, g x_{n+1}\right), \\
& \left.\left(d\left(g x_{n+1}, g x_{n+1}\right) \cdot d\left(g x_{n+2}, g x_{n}\right)\right)^{1 / 2}\right\} \\
& \leq \max \left\{d\left(g x_{n}, g x_{n+1}\right), d\left(g x_{n+2}, g x_{n+1}\right),\right. \\
& \left.\left(d\left(g x_{n+2}, g x_{n+1}\right) \cdot d\left(g x_{n+1}, g x_{n}\right)\right)^{1 / 2}\right\} \\
& \leq \max \left\{d\left(g x_{n}, g x_{n+1}\right), d\left(g x_{n+2}, g x_{n+1}\right),\right. \\
& \left.\frac{d\left(g x_{n+2}, g x_{n+1}\right)+d\left(g x_{n+1}, g x_{n}\right)}{2}\right\} \\
& =\max \left\{d\left(g x_{n}, g x_{n+1}\right), d\left(g x_{n+2}, g x_{n+1}\right)\right\} .
\end{aligned}
$$

If, for some $n, \max \left\{d\left(g x_{n}, g x_{n+1}\right), d\left(g x_{n+2}, g x_{n+1}\right)\right\}=$ $d\left(g x_{n+2}, g x_{n+1}\right)$, then $M_{f, g}\left(x_{n}, x_{n+1}\right)=d\left(g x_{n+2}, g x_{n+1}\right)$ and

$$
\begin{aligned}
\psi\left(d\left(g x_{n+1}, g x_{n+2}\right)\right) & \leq \frac{\psi\left(M_{f, g}\left(x_{n}, x_{n+1}\right)\right)}{\varphi\left(M_{f, g}\left(x_{n}, x_{n+1}\right)\right)} \\
& \leq \frac{\psi\left(d\left(g x_{n+2}, g x_{n+1}\right)\right)}{\varphi\left(d\left(g x_{n+2}, g x_{n+1}\right)\right)}
\end{aligned}
$$

imply that $\varphi\left(d\left(g x_{n+2}, g x_{n+1}\right)\right)=1$, a contradiction as $d\left(g x_{n+2}, g x_{n+1}\right)>1$. Hence $d\left(g x_{n+2}, g x_{n+1}\right) \leq d\left(g x_{n}, g x_{n+1}\right)$ for all $n$. Thus $M_{f, g}\left(x_{n}, x_{n+1}\right) \leq d\left(g x_{n}, g x_{n+1}\right)$. On the other hand, we have $M_{f, g}\left(x_{n}, x_{n+1}\right) \geq d\left(g x_{n}, g x_{n+1}\right)$. Hence $M_{f, g}\left(x_{n}, x_{n+1}\right)=d\left(g x_{n}, g x_{n+1}\right)$.

Similarly we obtain that $d\left(g x_{n+1}, g x_{n}\right) \leq d\left(g x_{n}, g x_{n-1}\right)$. Thus the sequence $\left\{d\left(g x_{n+1}, g x_{n}\right)\right\}$ is nonincreasing. Consequently, there exists $\delta \geq 1$ such that $\lim _{n \rightarrow \infty} d\left(g x_{n+1}, g x_{n}\right)=$ $\delta$. Suppose that $\delta>1$. Now

$$
\psi\left(d\left(g x_{n+2}, g x_{n+1}\right)\right) \leq \frac{\psi\left(M_{f, g}\left(x_{n+1}, x_{n}\right)\right)}{\varphi\left(M_{f, g}\left(x_{n+1}, x_{n}\right)\right)},
$$

and lower semicontinuity of $\varphi$ gives that

$$
\begin{aligned}
& \limsup _{n \rightarrow \infty} \psi\left(d\left(g x_{n+2}, g x_{n+1}\right)\right) \\
& \quad \leq \frac{\lim \sup _{n \rightarrow \infty} \psi\left(M_{f, g}\left(x_{n+1}, x_{2 n}\right)\right)}{\lim \inf _{n \rightarrow \infty} \varphi\left(M_{f, g}\left(x_{2 n+1}, x_{2 n}\right)\right)},
\end{aligned}
$$

which implies that $\psi(\delta) \leq \psi(\delta) / \varphi(\delta)$, a contradiction. Therefore $\delta=1$. That is,

$$
\lim _{n \rightarrow \infty} d\left(g x_{n+1}, g x_{n}\right)=1 .
$$

Assume that $\left\{g x_{n}\right\}$ is not a multiplicative Cauchy sequence. Then, there is $\varepsilon>1$, and there are even integers $n_{k}$ and $m_{k}$ with $m_{k}>n_{k}>k$ such that

$$
d\left(g x_{m_{k}}, g x_{n_{k}}\right) \geq \varepsilon
$$

and $d\left(g x_{m_{k}-1}, g x_{n_{k}}\right)<\varepsilon$. Note that

$$
\begin{aligned}
\varepsilon & \leq d\left(g x_{m_{k}}, g x_{n_{k}}\right) \\
& \leq d\left(g x_{n_{k}}, g x_{m_{k}-1}\right) \cdot d\left(g x_{m_{k}-1}, g x_{m_{k}}\right) .
\end{aligned}
$$

From (39) and (40), it follows that

$$
\lim _{k \rightarrow \infty} d\left(g x_{m_{k}}, g x_{n_{k}}\right)=\varepsilon
$$

By (42) and

$$
\begin{array}{r}
d\left(g x_{n_{k}+1}, g x_{m_{k}}\right) \leq \\
d\left(g x_{n_{k}+1}, g x_{n_{k}}\right) \\
\cdot d\left(g x_{n_{k}}, g x_{m_{k}}\right),
\end{array}
$$

we have $\lim _{k \rightarrow \infty} d\left(g x_{n_{k}+1}, g x_{m_{k}}\right) \leq \varepsilon$. Also, by (42) and

$$
d\left(g x_{m_{k}}, g x_{n_{k}}\right) \leq d\left(g x_{n_{k}+1}, g x_{n_{k}}\right) \cdot d\left(g x_{n_{k}+1}, g x_{m_{k}}\right)
$$

we obtain that $\varepsilon \leq \lim _{k \rightarrow \infty} d\left(g x_{n_{k}+1}, g x_{m_{k}}\right)$. Hence

$$
\lim _{k \rightarrow \infty} d\left(g x_{n_{k}+1}, g x_{m_{k}}\right)=\varepsilon
$$

From (39) and

$$
\begin{aligned}
d\left(g x_{n_{k}+1}, g x_{m_{k}-1}\right) \leq & d\left(g x_{n_{k}+1}, g x_{n_{k}}\right) \\
& \cdot d\left(g x_{n_{k}}, g x_{m_{k}-1}\right),
\end{aligned}
$$

we have $\lim _{k \rightarrow \infty} d\left(g x_{n_{k}+1}, g x_{m_{k}-1}\right) \leq \varepsilon$. By (45) and the inequality

$$
\begin{array}{r}
d\left(g x_{n_{k}+1}, g x_{m_{k}}\right) \leq \\
\cdot d\left(g x_{n_{k}+1}, g x_{m_{k}-1}\right) \\
\cdot d\left(g x_{m_{k}-1}, g x_{m_{k}}\right),
\end{array}
$$

we have $\varepsilon \leq \lim _{k \rightarrow \infty} d\left(g x_{n_{k}+1}, g x_{m_{k}-1}\right)$. Thus

$$
\lim _{k \rightarrow \infty} d\left(g x_{n_{k}+1}, g x_{m_{k}-1}\right)=\varepsilon
$$

Note that

$$
\begin{aligned}
& M_{f, g}\left(x_{n_{k}}, x_{m_{k}-1}\right)=\max \left\{d\left(f x_{n_{k}}, f x_{m_{k}-1}\right),\right. \\
& d\left(f x_{n_{k}}, g x_{n_{k}}\right), d\left(f x_{m_{k}-1}, g x_{m_{k}-1}\right), \\
& \left.\left(d\left(f x_{n_{k}}, g x_{m_{k}-1}\right) \cdot d\left(f x_{m_{k}-1}, g x_{n_{k}}\right)\right)^{1 / 2}\right\} \\
& =\max \left\{d\left(g x_{n_{k}+1}, g x_{m_{k}}\right), d\left(g x_{n_{k}+1}, g x_{n_{k}}\right),\right. \\
& d\left(g x_{m_{k}}, g x_{m_{k}-1}\right), \\
& \left.\left(d\left(g x_{n_{k}+1}, g x_{m_{k}-1}\right) \cdot d\left(g x_{m_{k}}, g x_{n_{k}}\right)\right)^{1 / 2}\right\} .
\end{aligned}
$$


Consequently, $\lim _{k \rightarrow \infty} M_{f, g}\left(x_{n_{k}}, x_{m_{k}-1}\right)=\max \{\varepsilon, 1,1, \varepsilon\}=\varepsilon$. Since $x_{n_{k}}$ and $x_{m_{k}-1}$ lie in different but adjacent labelled sets $A_{i}$ and $A_{i+1}$ for some $1 \leq i \leq p$, we have

$$
\begin{aligned}
\psi\left(d\left(g x_{n_{k}+1}, g x_{m_{k}}\right)\right) & =\psi\left(d\left(f x_{n_{k}}, g x_{m_{k}-1}\right)\right) \\
& \leq \frac{\psi\left(M_{f, g}\left(x_{n_{k}}, x_{m_{k}-1}\right)\right)}{\varphi\left(M_{f, g}\left(x_{n_{k}}, x_{m_{k}-1}\right)\right)} .
\end{aligned}
$$

Taking upper limit as $k \rightarrow \infty$ implies that $\psi(\varepsilon) \leq \psi(\varepsilon) / \varphi(\varepsilon)$, a contradiction as $\varepsilon>1$, and hence $\left\{g x_{n}\right\}$ is a multiplicative Cauchy sequence in $\cup_{i=1}^{p} g\left(A_{i}\right)$. Since $\cup_{i=1}^{p} g\left(A_{i}\right)$ is complete, there exists $y \in \cup_{i=1}^{p} g\left(A_{i}\right)$ such that

$$
\lim _{k \rightarrow \infty} d\left(g x_{n}, y\right)=1
$$

Consequently, we can find a point $z$ in $Y$ such that $g(z)=y$.

Now we show that $y \in \cap_{i=1}^{p} g\left(A_{i}\right)=Z$. From condition (a) and $x_{0} \in A_{i_{0}}$ for some $i_{0} \in\{1,2, \ldots, p\}$, we can choose a subsequence $\left\{x_{n_{k}}\right\}$ in $A_{i_{0}}$ out of the sequence $\left\{x_{n}\right\}$. Obviously, $\left\{g x_{n_{k}}\right\} \subseteq g\left(A_{i_{0}}\right)$. As $g\left(A_{i_{0}}\right)$ is closed, $y \in g\left(A_{i_{0}}\right)$. Similarly, we can choose a subsequence $\left\{x_{n_{k}+1}\right\}$ in $A_{i_{0}+1}$ out of the sequence $\left\{x_{n}\right\}$. Obviously, $\left\{g x_{n_{k}+1}\right\} \subseteq g\left(A_{i_{0}+1}\right)$. As $g\left(A_{i_{0}+1}\right)$ is closed, $y \in g\left(A_{i_{0}+1}\right)$. Continuing this way, we obtain that $y \in \cap_{i=1}^{p} g\left(A_{i}\right)$ and hence $\cap_{i=1}^{p} g\left(A_{i}\right) \neq \emptyset$.

Now we show that $f(z)=g(z)$. Since $z \in Y$, there exists some $i$ in $\{1,2, \ldots, p\}$ such that $z \in A_{i}$. Choose a subsequence $\left\{x_{n_{k}}\right\}$ of $\left\{x_{n}\right\}$ with $x_{n_{k}} \in A_{i-1}$. From (b), we have

$$
\begin{aligned}
\psi\left(d\left(g x_{n_{k}+1}, f z\right)\right) & =\psi\left(d\left(f x_{n_{k}}, f z\right)\right) \\
& \leq \frac{\psi\left(M_{f, g}\left(x_{n_{k}}, z\right)\right)}{\varphi\left(M_{f, g}\left(x_{n_{k}}, z\right)\right)},
\end{aligned}
$$

where

$$
\begin{aligned}
& M_{f, g}\left(x_{n_{k}}, z\right)=\max \left\{d\left(g x_{n_{k}}, g z\right), d\left(f x_{n_{k}}, g x_{n_{k}}\right),\right. \\
& \left.d(f z, g z),\left(d\left(f x_{n_{k}}, g z\right) \cdot d\left(f z, g x_{n_{k}}\right)\right)^{1 / 2}\right\} \\
& =\max \left\{d\left(g x_{n_{k}}, g z\right), d\left(g x_{n_{k}+1}, g x_{n_{k}}\right), d(f z, g z),\right. \\
& \left.\left(d\left(g x_{n_{k}+1}, g z\right) \cdot d\left(f z, g x_{n_{k}}\right)\right)^{1 / 2}\right\} .
\end{aligned}
$$

On taking upper limit as $k \rightarrow \infty$ we obtain that

$$
\psi(d(g z, f z)) \leq \frac{\psi(d(f z, g z))}{\varphi(d(f z, g z))},
$$

and hence $f(z)=g(z)$. Thus $z$ is the coincidence point of $f$ and $g$.

Note that $Z$ being a finite intersection of closed sets is closed and hence complete. Consider the restrictions of $f$ and $g$ on $Z$; that is, $\left.f\right|_{Z},\left.g\right|_{Z}: Z \rightarrow Z$. Obviously, $\left.f\right|_{Z}(Z) \subseteq$ $\left.g\right|_{Z}(Z) \subseteq Z$. Also $\left.g\right|_{Z}(Z)$ is closed and hence complete. From Theorem 18, it follows that $\left.f\right|_{Z}$ and $\left.g\right|_{Z}$ have a unique coincidence point in $Z$. As $f$ and $g$ are weakly compatible, from Proposition 12, it follows that $\left.f\right|_{Z}$ and $\left.g\right|_{Z}$ have a unique common fixed point.

Corollary 20. Let $(X, d)$ be a multiplicative metric space, $A_{1}, A_{2}, \ldots, A_{p}$ nonempty closed subsets of $X$, and $Y=\cup_{i=1}^{P} A_{i}$. Suppose that $f, g: Y \rightarrow Y$ are such that

(a) $Y$ has a cocyclic representation with respect to pair $\left(f^{m}, g^{n}\right)$ and the collection $\left\{A_{i}: i=1,2, \ldots, p\right\}$ for some $m, n \in \mathbb{N}$;

(b) there exists two control functions $\psi$ and $\varphi$ such that, for any $(x, y) \in A_{i} \times A_{i+1}, i=1,2, \ldots, p$,

$$
\psi\left(d\left(f^{m} x, f^{m} y\right)\right) \leq \frac{\psi\left(M_{f, g}(x, y)\right)}{\varphi\left(M_{f, g}(x, y)\right)}
$$

holds for some $m, n \in \mathbb{N}$, where

$$
\begin{aligned}
& M_{f, g}(x, y)=\max \left\{d\left(g^{n} x, g^{n} y\right), d\left(f^{m} x, g^{n} x\right),\right. \\
& \left.d\left(f^{m} y, g^{n} y\right),\left(d\left(f^{m} x, g^{n} y\right) \cdot d\left(f^{m} y, g^{n} x\right)\right)^{1 / 2}\right\}
\end{aligned}
$$

$$
\text { with } A_{p+1}=A_{1} \text {. }
$$

If $g^{n}\left(A_{i}\right)$ is complete subspace of $X$ for each $i \in$ $\{1,2, \ldots, p\}$, then $f^{m}$ and $g^{n}$ have a unique coincidence point $z \in \cap_{i=1}^{p} g^{n}\left(A_{i}\right)=Z$ provided that $f^{m}(Z) \subseteq g^{n}(Z) \subseteq$ $Z$ and $g^{n}(Z)$ is closed. Moreover, if $f^{m}$ and $g^{n}$ are weakly compatible, then $f^{m}$ and $g^{n}$ have a unique common fixed point. Furthermore, $f$ and $g$ have a unique common fixed point provided that $f$ and $g$ are commuting.

Proof. Set $S=f^{m}$ and $T=g^{n}$. From Theorem 19, $S$ and $T$ have a unique common fixed point $u$. Now $S(f u)=f(S u)=$ $f u$ and $T(f u)=f(T u)=f u$ imply that $f u$ is the common fixed point of $S$ and $T$. Also, $T(g u)=g(T u)=g u$ and $S(g u)=$ $g(S u)=g u$ imply that $g u$ is also the common fixed point of $S$ and $T$. By uniqueness of common fixed point of $S$ and $T$, we have $f u=g u=u$.

Example 21. Let $X=\mathbb{R}$ and let $d$ be a multiplicative metric on $X$ defined by $d(x, y)=a^{|x-y|}$, where $a>1$ is a real number. For some $c>1$, set $A_{1}=[-c, 0], A_{2}=[0, c]$, and $A_{3}=A_{1}$. Define $f, g: \cup_{i=1}^{2} A_{i} \rightarrow \cup_{i=1}^{2} A_{i}$ by

$$
\begin{aligned}
& f(x)=\frac{\alpha x}{c}, \\
& g(x)=-\frac{\beta x}{c},
\end{aligned}
$$

where $\alpha, \beta \geq 1$ with $6 \alpha \leq 5 \beta$ and $\beta \leq c$. Note that $f\left(A_{1}\right)=$ $[-\alpha, 0] \subseteq[-\beta, 0]=g\left(A_{2}\right)$ and $f\left(A_{2}\right)=[0, \alpha] \subseteq[0, \beta]=$ $g\left(A_{1}\right) . Y=A_{1} \cup A_{2}$ has a cocyclic representation with respect to pair $(f, g)$ and the collection $\left\{A_{1}, A_{2}\right\}$. 
Define $\psi, \varphi:[1, \infty) \rightarrow[1, \infty)$ by

$$
\begin{aligned}
& \psi(t)=t^{3}, \\
& \varphi(t)= \begin{cases}t^{1 / 2}, & \text { if } t \in[1,2 \beta], \\
t^{2}, & \text { if } t>2 \beta\end{cases}
\end{aligned}
$$

Clearly $\psi$ is continuous and nondecreasing, $\varphi$ is a lower semicontinuous, and $\psi(t)=\varphi(t)=1$ if and only if $t=1$.

We show that condition (b) is satisfied. Now, for $x \in A_{1}$, $y \in A_{2}$ implies

$$
\begin{aligned}
\psi(d(f x, f y)) & =\psi\left(d\left(\frac{\alpha x}{c}, \frac{\alpha y}{c}\right)\right)=\psi\left(a^{(\alpha / c)(y-x)}\right) \\
& =a^{(3 \alpha / c)(y-x)} \leq a^{(5 \beta / 2 c)(y-x)} \\
& =\left(d\left(-\frac{\beta x}{c},-\frac{\beta y}{c}\right)\right)^{5 / 2} \\
& =d(g x, g y)^{5 / 2} \leq\left[M_{f, g}(x, y)\right]^{5 / 2} \\
& =\frac{\left[M_{f, g}(x, y)\right]^{3}}{\left[M_{f, g}(x, y)\right]^{1 / 2}} \\
& =\frac{\psi\left(M_{f, g}(x, y)\right)}{\varphi\left(M_{f, g}(x, y)\right)} .
\end{aligned}
$$

When $x \in A_{2}$ and $y \in A_{1}$,

$$
\begin{aligned}
\psi(d(f x, f y)) & =\psi\left(d\left(\frac{\alpha x}{c}, \frac{\alpha y}{c}\right)\right)=\psi\left(a^{(\alpha / c)(x-y)}\right) \\
& =a^{(3 \alpha / c)(x-y)} \leq a^{(5 \beta / 2 c)(x-y)} \\
& =\left(d\left(-\frac{\beta x}{c},-\frac{\beta y}{c}\right)\right)^{5 / 2} \\
& =d(g x, g y)^{5 / 2} \leq\left[M_{f, g}(x, y)\right]^{5 / 2} \\
& =\frac{\left[M_{f, g}(x, y)\right]^{3}}{\left[M_{f, g}(x, y)\right]^{1 / 2}} \\
& =\frac{\psi\left(M_{f, g}(x, y)\right)}{\varphi\left(M_{f, g}(x, y)\right)} .
\end{aligned}
$$

Thus, $f$ and $g$ satisfy all the conditions of Theorem 19 . Moreover, $f$ and $g$ have at most one common fixed point.

Example 22. Let $X=\mathbb{R}$ and $d: X \times X \rightarrow \mathbb{R}^{+}$be the multiplicative metric defined by $d(x, y)=e^{|x-y|}$. Suppose
$A_{1}=[-c, 0], A_{2}=[-c / 2, c / 2]$, and $A_{3}=[-c / 4, c]$ and $A_{4}=A_{1}$, where $c>0$. Define $f, g: \cup_{i=1}^{3} A_{i} \rightarrow \cup_{i=1}^{3} A_{i}$ by

$$
\begin{aligned}
& f x= \begin{cases}-\frac{x}{c} & \text { if } x>0, \\
0 & \text { otherwise, }\end{cases} \\
& g x=\frac{2 x}{c} .
\end{aligned}
$$

Note that $f\left(A_{1}\right)=\{0\} \subseteq[-1,1]=g\left(A_{2}\right), f\left(A_{2}\right)=$ $[-1 / 2,0] \subseteq[-1 / 2,2]=g\left(A_{3}\right)$, and $f\left(A_{3}\right)=[-1,0] \subseteq$ $[-2,0]=g\left(A_{1}\right)$. Hence $Y=A_{1} \cup A_{2}$ is a cocyclic representation between $f$ and $g$.

Let $\psi, \varphi:[1, \infty) \rightarrow[1, \infty)$ be as defined in Example 21. To check condition (b), we consider the following cases:

(1) Let $x \in A_{1}$ and $y \in A_{2}$. If $y \in[-c / 2,0]$, then $d(f x, f y)=e^{0}=1$ and hence (b) holds. If $y \in$ $(0, c / 2]$, then we have

$$
\begin{aligned}
d(f x, f y) & =d\left(0,-\frac{y}{c}\right)=e^{(1 / c) y} \leq e^{(5 / c)(y-x)} \\
& =d\left(\frac{2 x}{c}, \frac{2 y}{c}\right)^{5 / 2}=d(g x, g y)^{5 / 2} \\
& \leq\left[M_{f, g}(x, y)\right]^{5 / 2}=\frac{\left[M_{f, g}(x, y)\right]^{3}}{\left[M_{f, g}(x, y)\right]^{1 / 2}} \\
& =\frac{\psi\left(M_{f, g}(x, y)\right)}{\varphi\left(M_{f, g}(x, y)\right)} .
\end{aligned}
$$

(2) Let $x \in A_{2}$ and $y \in A_{3}$. If $x \in[-c / 2,0]$ and $y \in$ $[-c / 4,0]$, then $d(f x, f y)=e^{0}=1$ and hence $(\mathrm{b})$ is satisfied. If $x \in[-c / 2,0]$ and $y \in(0, c]$, then we obtain that

$$
\begin{aligned}
d(f x, f y) & =d\left(0,-\frac{y}{c}\right)=e^{(1 / c) y}<e^{(15 / 2 c) y} \\
& =d\left(-\frac{y}{c}, \frac{2 y}{c}\right)^{5 / 2}=d(f y, g y)^{5 / 2} \\
& \leq\left[M_{f, g}(x, y)\right]^{5 / 2}=\frac{\left[M_{f, g}(x, y)\right]^{3}}{\left[M_{f, g}(x, y)\right]^{1 / 2}} \\
& =\frac{\psi\left(M_{f, g}(x, y)\right)}{\varphi\left(M_{f, g}(x, y)\right)} .
\end{aligned}
$$

When $x \in(0, c / 2]$ and $y \in(-c / 4,0]$, we have

$$
\begin{aligned}
d(f x, f y) & =d\left(-\frac{x}{c}, 0\right)=e^{(1 / c) x}<e^{(15 / 2 c) x} \\
& =d\left(-\frac{x}{c}, \frac{2 x}{c}\right)^{5 / 2}=d(f x, g x)^{5 / 2}
\end{aligned}
$$




$$
\begin{aligned}
& \leq\left[M_{f, g}(x, y)\right]^{5 / 2}=\frac{\left[M_{f, g}(x, y)\right]^{3}}{\left[M_{f, g}(x, y)\right]^{1 / 2}} \\
& =\frac{\psi\left(M_{f, g}(x, y)\right)}{\varphi\left(M_{f, g}(x, y)\right)} .
\end{aligned}
$$

For $x \in(0, c / 2]$ and $y \in(0, c]$, we obtain that

$$
\begin{aligned}
d(f x, f y) & =d\left(-\frac{x}{c},-\frac{y}{c}\right)=e^{(1 / c)|x-y|}<e^{(5 / c)|x-y|} \\
& =d\left(\frac{2 x}{c}, \frac{2 y}{c}\right)^{5 / 2}=d(g x, g y)^{5 / 2} \\
& \leq\left[M_{f, g}(x, y)\right]^{5 / 2}=\frac{\left[M_{f, g}(x, y)\right]^{3}}{\left[M_{f, g}(x, y)\right]^{1 / 2}} \\
& =\frac{\psi\left(M_{f, g}(x, y)\right)}{\varphi\left(M_{f, g}(x, y)\right)} .
\end{aligned}
$$

(3) Let $x \in A_{3}$ and $y \in A_{1}$. If $x \in[-c / 4,0]$, then $d(f x, f y)=1$ and (b) is satisfied. If $x \in(0, c]$, then we have

$$
\begin{aligned}
d(f x, f y) & =d\left(-\frac{x}{c}, 0\right)=e^{(1 / c) x}<e^{(15 / 2 c) x} \\
& =d\left(-\frac{x}{c}, \frac{2 x}{c}\right)^{5 / 2}=d(f x, g x)^{5 / 2} \\
& \leq\left[M_{f, g}(x, y)\right]^{5 / 2}=\frac{\left[M_{f, g}(x, y)\right]^{3}}{\left[M_{f, g}(x, y)\right]^{1 / 2}} \\
& =\frac{\psi\left(M_{f, g}(x, y)\right)}{\varphi\left(M_{f, g}(x, y)\right)} .
\end{aligned}
$$

Hence $f$ and $g$ satisfy all the conditions of Theorem 19 . Moreover, 0 is a unique common fixed point of $f$ and $g$ in $\cap_{i=1}^{3} A_{i}$.

Denote $\Upsilon=\{\phi:[1, \infty) \rightarrow[1, \infty) ; \phi$ is a Lebesgue integrable with finite integral on each compact subset of $[1, \infty)$ and for each $\left.\varepsilon>1, \int_{1}^{\varepsilon} \varphi(t) d t>1\right\}$.

As a consequence of Theorem 19, we obtain following common fixed point result for mappings satisfying cocyclic contractive condition of integral type in a multiplicative metric space.

Corollary 23. Let $(X, d)$ be a multiplicative metric space, $A_{1}, A_{2}, \ldots, A_{p}, p$ nonempty closed subsets of $X$, and $Y=$ $\cup_{i=1}^{p} A_{i}$. Suppose that $f, g: Y \rightarrow Y$ are mappings such that

(a) $Y$ has a cocyclic representation with respect to pair $(f, g)$ and the collection $\left\{A_{i}: i=1,2, \ldots, p\right\}$; (b) there exists control functions $\psi$ and $\varphi$ such that, for any $(x, y) \in A_{i} \times A_{i+1}, i=1,2, \ldots, p$, the following,

$$
\int_{1}^{\psi(d(f x, f y))} \phi(t) d t \leq \frac{\int_{1}^{\psi\left(M_{f, g}(x, y)\right)} \phi(t) d t}{\int_{1}^{\varphi\left(M_{f, g}(x, y)\right)} \phi(t) d t},
$$

is satisfied, where $\phi \in \Upsilon$, and

$$
\begin{gathered}
M_{f, g}(x, y)=\max \{d(g x, g y), d(f x, g x), \\
\left.d(f y, g y),(d(f x, g y) \cdot d(f y, g x))^{1 / 2}\right\}
\end{gathered}
$$

with $A_{p+1}=A_{1}$.

If $g\left(A_{i}\right)$ is a complete subspace of $X$ for each $i \in$ $\{1,2, \ldots, p\}$, then $f$ and $g$ have a unique coincidence point $z \in \cap_{i=1}^{p} g\left(A_{i}\right)=Z$ provided that $f(Z) \subseteq g(Z) \subseteq Z$ and $g(Z)$ is closed. Moreover, if $f$ and $g$ are weakly compatible, then $f$ and $g$ have at most one common fixed point.

Proof. Define $\Psi:[1, \infty) \rightarrow[1, \infty)$ by $\Psi(x)=\int_{1}^{x} \phi(t) d t$. From (67), it follows that

$$
\Psi(\psi(d(f x, f y))) \leq \frac{\Psi\left(\psi\left(M_{f, g}(x, y)\right)\right)}{\Psi\left(\varphi\left(M_{f, g}(x, y)\right)\right)},
$$

which can be written as

$$
\psi_{1}(d(f x, f y)) \leq \frac{\psi_{1}\left(M_{f, g}(x, y)\right)}{\varphi_{1}(M(x, y))},
$$

where $\psi_{1}=\Psi \circ \psi$ and $\varphi_{1}=\Psi \circ \varphi$. Clearly, $\psi_{1}, \varphi_{1}:[1, \infty) \rightarrow$ $[1, \infty), \psi_{1}$ is continuous and nondecreasing, $\varphi_{1}$ is a lower semicontinuous, and $\psi_{1}(t)=\varphi_{1}(t)=1$ if and only if $t=1$. Hence by Theorem 19, $f$ and $g$ have at most one common fixed point.

\section{Conflict of Interests}

The authors declare that there is no conflict of interests regarding the publication of this paper.

\section{Acknowledgments}

The second author thanks the Spanish Ministry of Economy and Competitiveness for its partial support of this work through Grant DPI2012-30651. The authors also thank the Basque Government for its support through Grant IT378-10 and the University of Basque Country for its support through Grant UFI 11/07.

\section{References}

[1] Y. I. Alber and S. Guerre-Delabriere, "Principle of weakly contractive maps in Hilbert space," in New Results in Operator Theory, Advances and Applications, I. Gohberg and Y. Lyubich, Eds., vol. 98, pp. 7-22, Birkhäuser, Basel, Switzerland, 1997. 
[2] I. Beg and M. Abbas, "Coincidence point and invariant approximation for mappings satisfying generalized weak contractive condition," Fixed Point Theory and Applications, vol. 2006, Article ID 74503, 7 pages, 2006.

[3] E. Karapinar, "Fixed point theory for cyclic weak $\phi$ contraction," Applied Mathematics Letters, vol. 24, pp. 822-825, 2011.

[4] W. A. Kirk, P. S. Srinivasan, and P. Veeramani, "Fixed points for mappings satisfying cyclical contractive conditions," Fixed Point Theory, vol. 4, no. 1, pp. 79-89, 2003.

[5] B. E. Rhoades, "Some theorems on weakly contractive maps," Nonlinear Analysis, vol. 47, no. 4, pp. 2683-2693, 2001.

[6] M. Păcurar and I. A. Rus, "Fixed point theory for cyclic $\phi$ contractions," Nonlinear Analysis: Theory, Methods \& Applications, vol. 72, no. 3-4, pp. 1181-1187, 2010.

[7] B. Piątek, "On cyclic Meir-Keeler contractions in metric spaces," Nonlinear Analysis: Theory, Methods \& Applications, vol. 74, no. 1, pp. 35-40, 2011.

[8] M. Derafshpour, S. Rezapour, and N. Shahzad, "On the existence of best proximity points of cyclic contractions," Advances in Dynamical Systems and Applications, vol. 6, no. 1, pp. 33-40, 2011.

[9] M. Abbas, T. Nazir, and S. Romaguera, "Fixed point results for generalized cyclic contraction mappings in partial metric spaces," Revista de la Real Academia de Ciencias Exactas, Fisicas y Naturales. Serie A. Matematicas, vol. 106, no. 2, pp. 287-297, 2012.

[10] R. P. Agarwal, M. A. Alghamdi, and N. Shahzad, "Fixed point theory for cyclic generalized contractions in partial metric spaces," Fixed Point Theory and Applications, vol. 2012, article 40, 2012.

[11] H. Aydi, C. Vetro, W. Sintunavarat, and P. Kumam, "Coincidence and fixed points for contractions and cyclical contractions in partial metric spaces," Fixed Point Theory and Applications, vol. 2012, article 124, 2012.

[12] P. Chaipunya, Y. J. Cho, W. Sintunavarat, and P. Kumam, "Fixed point and common fixed point theorems for cyclic quasicontractions in metric and ultrametric spaces," Advances in Pure Mathematics, vol. 2, pp. 401-407, 2012.

[13] H. K. Nashine, W. Sintunavarat, and P. Kumam, "Cyclic generalized contractions and fixed point results with applications to an integral equation," Fixed Point Theory and Applications, vol. 2012, article 217, 13 pages, 2012.

[14] I. A. Rus, "Cyclic representation and fixed points," Annals of the Tiberiu Popoviciu Seminar of Functional Equations, Approximation and Convexity, vol. 3, pp. 171-178, 2005.

[15] W. Sintunavarat and P. Kumam, "Common fixed point theorem for cyclic generalized multi-valued contraction mappings," Applied Mathematics Letters, vol. 25, no. 11, pp. 1849-1855, 2012.

[16] M. Ozavsar and A. C. Cevikel, "Fixed points of multiplicative contraction mappings on multiplicative metric spaces," http://arxiv.org/abs/1205.5131.

[17] A. E. Bashirov, E. M. Kurpınar, and A. Özyapıc1, "Multiplicative calculus and its applications," Journal of Mathematical Analysis and Applications, vol. 337, no. 1, pp. 36-48, 2008.

[18] A. E. Bashirov, E. Misırlı, Y. Tandoğdu, and A. Özyapıcı, "On modeling with multiplicative differential equations," Applied Mathematics-A Journal of Chinese Universities, vol. 26, no. 4, pp. 425-438, 2011.

[19] L. Florack and H. van Assen, "Multiplicative calculus in biomedical image analysis," Journal of Mathematical Imaging and Vision, vol. 42, no. 1, pp. 64-75, 2012.
[20] X. He, M. Song, and D. Chen, "Common fixed points for weak commutative mappings on a multiplicative metric space," Fixed Point Theory and Applications, vol. 2014, article 48, 2014.

[21] M. Abbas, B. Ali, and Y. I. Suleiman, "Common fixed points of locally contractive mappings in multiplicative metric spaces with application," International Journal of Mathematics and Mathematical Sciences, vol. 2015, Article ID 218683, 7 pages, 2015.

[22] O. Yamaod and W. Sintunavarat, "Some fixed point results for generalized contraction mappings with cyclic $(\alpha, \beta)$-admissible mapping in multiplicative metric spaces," Journal of Inequalities and Applications, vol. 2014, article 488, 15 pages, 2014.

[23] M. Abbas and G. Jungck, "Common fixed point results for noncommuting mappings without continuity in cone metric spaces," Journal of Mathematical Analysis and Applications, vol. 341, no. 1, pp. 416-420, 2008.

[24] R. H. Haghi, Sh. Rezapour, and N. Shahzad, "Some fixed point generalizations are not real generalizations," Nonlinear Analysis: Theory, Methods \& Applications, vol. 74, no. 5, pp. 1799-1803, 2011. 


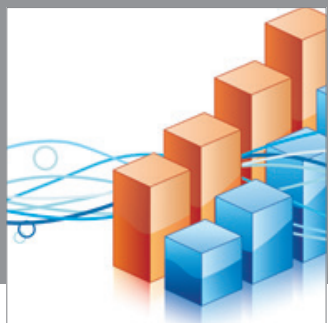

Advances in

Operations Research

mansans

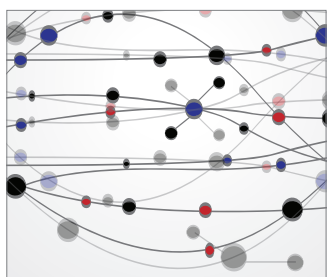

The Scientific World Journal
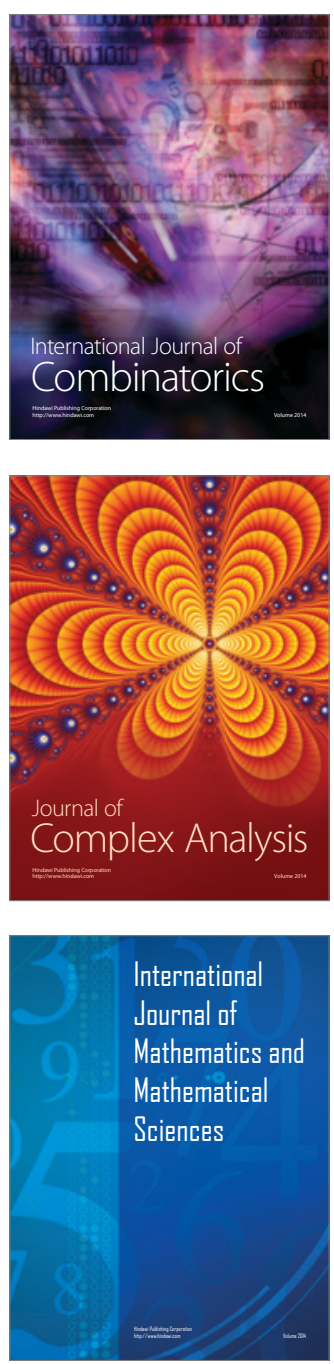
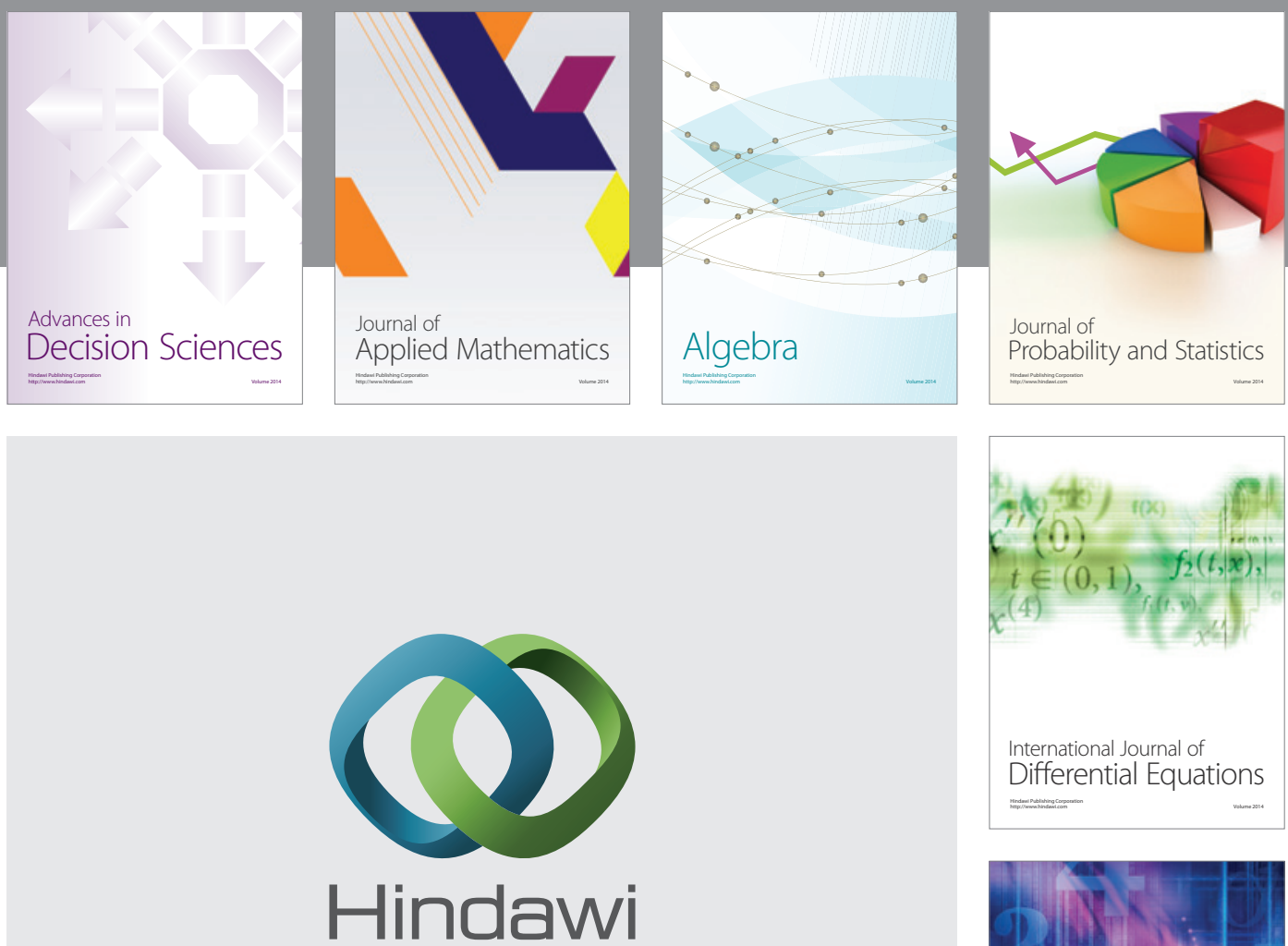

Submit your manuscripts at http://www.hindawi.com
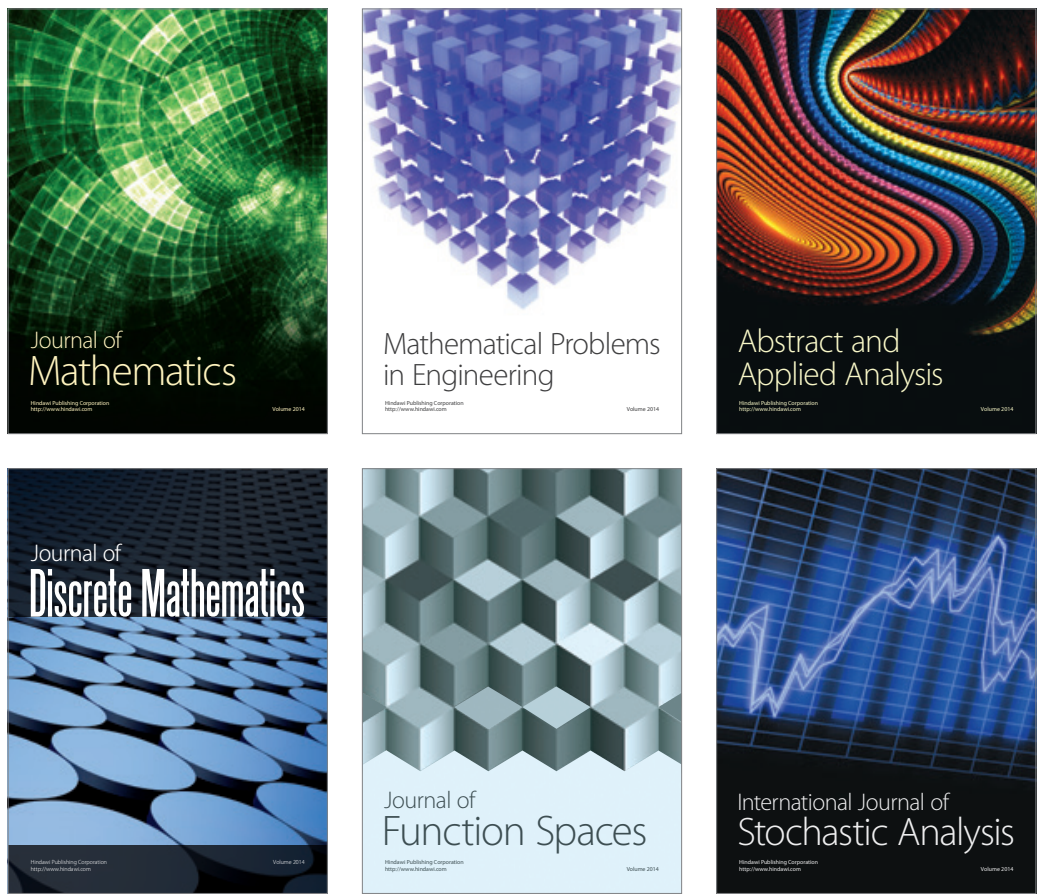

Journal of

Function Spaces

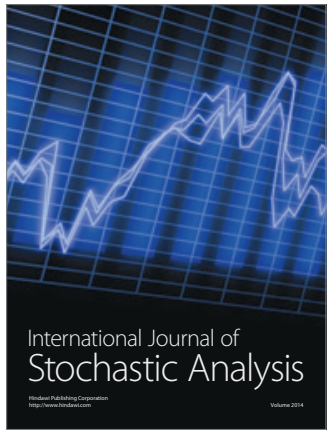

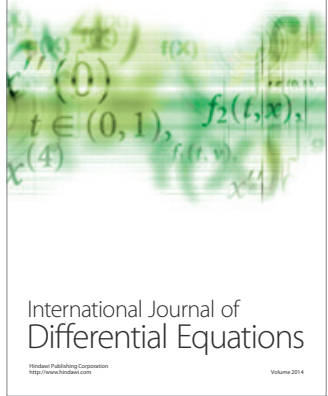
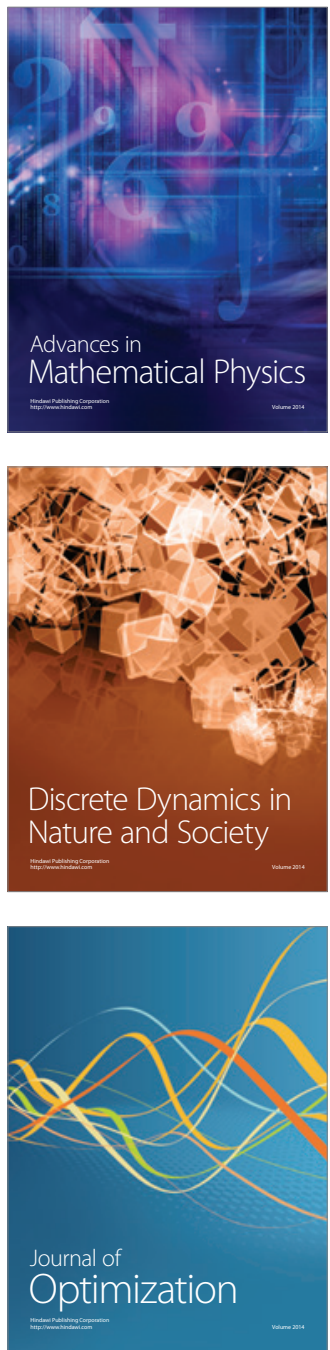